\title{
Research
}

\section{Pandemic influenza A(H1N1)pdm09:}

\author{
risk of infection in primary healthcare workers
}

\begin{abstract}
Background

Healthcare workers in primary care are at risk of infection during an influenza pandemic. The 2009 influenza pandemic provided an opportunity to assess this risk.

\section{Aim}

To measure the prevalence of seropositivity to influenza A(H1N1)pdm09 among primary healthcare workers in Canterbury, New Zealand, following the 2009 influenza pandemic, and to examine associations between seropositivity and participants' sociodemographic characteristics, professional roles, work patterns, and seasonal influenza vaccination status.
\end{abstract}

\section{Design and setting}

An observational study involving a questionnaire and testing for influenza A(H1N1)pdm09 seropositivity in all primary healthcare workers in Canterbury, New Zealand between December 2009 and February 2010.

\section{Method}

Participants completed a questionnaire that recorded sociodemographic and professional data, symptoms of influenza-like illness, history of seasonal influenza vaccination, and work patterns. Serum samples were collected and haemagglutination inhibition antibody titres to influenza A(H1N1)pdm09 measured.

\section{Results}

Questionnaires and serum samples were received from 1027 participants, from a workforce of 1476 (response rate 70\%). Seropositivity was detected in 224 participants (22\%). Receipt of seasonal influenza vaccine lodds ratio $[\mathrm{OR}]=2.0,95 \%$ confidence interval $[\mathrm{Cl}]=1.2$ to 3.3$)$, recall of influenza $(\mathrm{OR}=1.9,95 \%$ $\mathrm{Cl}=1.3$ to 2.8$)$, and age $\leq 45$ years $(\mathrm{OR}=1.4,95 \%$ $\mathrm{Cl}=1.0$ to 1.9 ) were associated with seropositivity.

\section{Conclusion}

A total of $22 \%$ of primary care healthcare workers were seropositive. Younger participants, those who recalled having influenza, and those who had been vaccinated against seasonal influenza were more likely to be seropositive. Working in a dedicated influenza centre was not associated with an increased risk of seropositivity.

\section{Keywords}

influenza A virus, H1N1 subtype; pandemics; primary care; vaccination

\section{INTRODUCTION}

The pandemic influenza A(H1N1)pdm09 virus emerged in Mexico in March 2009, and the World Health Organization (WHO) declared a global influenza pandemic on 11 June 2009.2 The virus arrived in New Zealand in April 2009, and the incidence of influenza increased rapidly from June, peaking in July (Figure 1). ${ }^{3}$ During the pandemic period in New Zealand, 1122 patients were admitted to hospital with influenza $\mathrm{A}(\mathrm{H} 1 \mathrm{~N} 1) \mathrm{pdm} 09$ and there were 49 influenza $\mathrm{A}(\mathrm{H} 1 \mathrm{~N} 1) p d m 09$-associated deaths. 4.5

Health planners in Canterbury (New Zealand's second largest geographic region) had developed a health-system pandemic response and this was triggered in April 2009.6 A key component of this response was the direction of probable influenza sufferers who needed medical attention to community-based assessment centres, rather than to their usual GP, in an attempt to keep health centres free of influenza. ${ }^{6}$ Community-based assessment centres were staffed by GPs, practice nurses, hospital nurses and doctors, and administrative staff. These workers were provided with personal protective equipment (surgical scrubs, disposable surgical gowns, masks, and gloves) to minimise the risk of infection, and they practised good infection control; however

B Hudson, MRCGP, FRNZCGP, senior lecturer in general practice; L Toop, MD (Brist), MRCGP FRNZCGP, professor of general practice;

D Mangin, DPH, FRNZCGP, associate professor of general practice; C Brunton, DComH, FAFPHM, FNZCPHM, senior lecturer; L Fletcher, BSc(Hons), MSc, DipStat, biostatistician, Department of Public Health and General Practice, University of Otago, Christchurch, New Zealand. L Jennings, PhD, FRCPath, FFSc(RCPA), clinical virologist, Microbiology Unit, Canterbury Health Laboratories and Department of Pathology, University of Otago, Christchurch, New Zealand. they are assumed to have had greater exposure to influenza than those who did not work at the community-based assessment centres. Higher rates of seroconversion have been reported previously among frontline hospital healthcare workers compared to hospital staff working in non-front-line positions, ${ }^{7}$ but it is not known whether primary care staff may also have been exposed to differential levels of risk.

Healthcare workers may be at greater risk of influenza infection than non-healthcare workers, and staff working in primary care, who have contact with patients early in the course of illness may be at greater risk of infection, since influenza viral shedding is maximal at this stage. 8.9 However, while healthcare workers appear to be at greater risk of serologically confirmed infection, they do not appear to be more likely to experience symptomatic infection, possibly because of greater exposure to multiple strains of influenza, which may confer cross-protection and reduce symptom severity. ${ }^{8}$ This may have implications for transmission of infection to vulnerable patients, and for healthcare worker vaccination policy. The design of this study allowed comparison of the proportions of asymptomatic and symptomatic infections.

The effect of prior vaccination against seasonal influenza (which did not include the $A(\mathrm{H} 1 \mathrm{~N} 1) p d m 09$ strain) on the risk of

\section{Address for correspondence}

Ben Hudson, Department of Public Health and General Practice, University of Otago, Christchurch, PO Box 4345, Christchurch 8140, New Zealand.

E-mail: ben.hudsondatago.ac.nz Submitted: 24 October 2012; Editor's response: 26 November 2012; final acceptance: 28 January 2013.

\section{CBritish Journal of General Practice}

This is the full-length article (published online 28 May 2013) of an abridged version published in print. Cite this article as: Br J Gen Pract 2013; DOI: 10.3399/bjgp13X668212 
Figure 1. Weekly consultation rates for influenzalike illness, all ages, New Zealand, 2007-2009. Reproduced with permission from Lopez $L$, Huang Q. Influenza in New Zealand 2009. Client report FW10019. Institute of Environmental Science and Research, 2010. ${ }^{4}$

\section{How this fits in \\ Healthcare workers working in front-line positions during an influenza pandemic may be at increased risk of infection, however, this risk has not been quantified in primary care. This study found no evidence of an increased risk of infection among healthcare workers working in a dedicated 'flu centre', and it appeared that primary healthcare workers were at no greater risk of infection than the general population. Healthcare workers who had received seasonal influenza vaccination, younger healthcare workers, and those who recalled an influenza-like illness were more likely to be seropositive to influenza A(H1N1)pdm09.}

infection with influenza A(H1N1)pdm09 is uncertain, and a range of estimates have been made, which range from indicating protection, ${ }^{10-13}$ to no effect, ${ }^{14-23}$ to increased susceptibility. ${ }^{24-26}$ A recent meta-analysis found that prior receipt of seasonal influenza vaccination may have been associated with moderate protection against influenza $\mathrm{A}(\mathrm{H} 1 \mathrm{~N} 1)$ pdm09. ${ }^{27}$ This study provides an opportunity to compare the risk of pandemic influenza infection among healthcare workers with and without a history of prior seasonal influenza vaccination.

This study aimed to measure the prevalence of seropositivity to influenza A(H1N1)pdm09 among primary healthcare workers in Canterbury, New Zealand, following the 2009 influenza pandemic, and to examine associations between seropositivity and participants sociodemographic characteristics, professional roles, work patterns, and seasonal influenza vaccination status.

\section{METHOD}

Sampling was conducted from December 2009 to February 2010. All GPs, practice nurses, and receptionists in Canterbury were invited to participate. All general practices in Canterbury were sent a set of study packs to be distributed to all GPs, practice nurses, and receptionists employed at each practice. The study packs included an information sheet, a consent form, a study questionnaire, identifying labels each with a unique study number, and a pre-printed laboratory request form.

The questionnaire recorded sociodemographic and professional variables, history of seasonal influenza vaccination, employment type within primary care, work patterns, and recall of influenza-like illness during the previous winter. All variables were recorded in precoded categories. Participants were also asked if they had experienced any of the following symptoms at any stage between 1 May and 31 October 2009: cough, sore

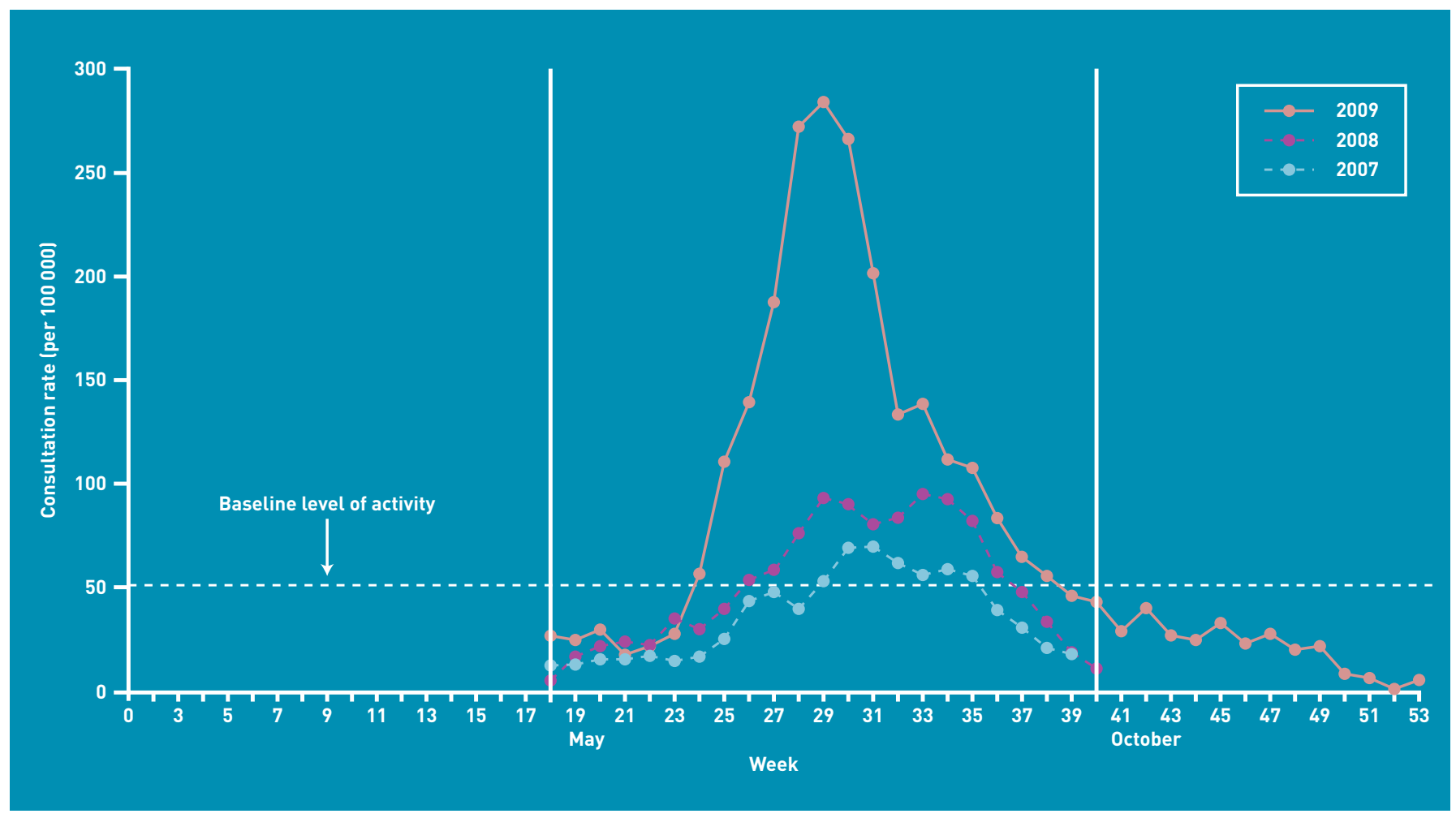


Table 1. Participant characteristics

\begin{tabular}{|c|c|c|c|c|}
\hline Characteristics & GPs, $n(\%)$ & Practice nurses, $n(\%)$ & Receptionists, $n(\%)$ & Total, $N(\%)$ \\
\hline \multicolumn{5}{|l|}{ Age, years ${ }^{a}$} \\
\hline$<25$ & $0(-)$ & $6(1.5)$ & $17(5.1)$ & $23(2.2)$ \\
\hline $25-35$ & $30(9.9)$ & $39(9.9)$ & $27(8.1)$ & 96 (9.3) \\
\hline $36-45$ & 89 (29.5) & 85 (21.7) & $56(16.9)$ & $230(22.4)$ \\
\hline $46-55$ & $121(40.1)$ & $163(41.6)$ & $120(36.1)$ & 404 (39.3) \\
\hline $56-65$ & 49 (16.2) & $84(21.4)$ & 98 (29.5) & $231(22.5)$ \\
\hline$>65$ & $13(4.3)$ & 15 (3.8) & $14(4.2)$ & $42(4.1)$ \\
\hline Female & $162(53.6)$ & $383(98.0)$ & 327 (98.2) & 872 (85.0) \\
\hline European & $245(81.1)$ & $359(91.6)$ & $301(90.4)$ & $905(88.1)$ \\
\hline Maori & $7(2.3)$ & 9 (2.3) & $10(3.0)$ & $26(2.5)$ \\
\hline Received seasonal flu vaccination ${ }^{a}$ & $270(89.7)$ & $324(82.9)$ & $277(83.2)$ & $871(85.0)$ \\
\hline Worked in community-based assessment centre ${ }^{b}$ & $107(35.9)$ & $71(18.2)$ & $23(7.0)$ & $201(19.7)$ \\
\hline Children aged $\leq 18$ years at home ${ }^{c}$ & $187(62.3)$ & $169(43.4)$ & $111(33.8)$ & $467(45.9)$ \\
\hline
\end{tabular}

throat, sneezing, fever or chills, runny or blocked nose, muscle aches or pains, and chest discomfort or breathing difficulties. Dummy variable coding was used for each symptom: one for experience of the symptom and zero otherwise.

Participants returned their completed questionnaires, labelled with their unique study number, and their consent forms, using reply-paid envelopes. Blood samples were drawn at the participants' own practice or at a community laboratory collection centre. Samples were also labelled with participants' unique study numbers and delivered to New Zealand's National Influenza Centre (NIC) for serological testing. Participants were able to access their own blood test results online, using their unique study number.

The NIC used an in-house haemagglutination inhibition assay developed and validated for measuring influenza A(H1N1)pdm09 antibodies. ${ }^{26}$ This test follows a WHO protocol and uses standardised virus preparation (4 haemagglutinating units per $25 \mu \mathrm{l}$ ), human sera treated with receptordestroying enzyme (RDE; Vibrio Cholera Neuraminidasel and guinea-pig red blood cells to remove non-specific inhibitors. The reciprocal of the highest dilution causing complete $(100 \%)$ haemagglutination inhibition is a measure of the antibody level to the $\mathrm{H} 1 \mathrm{~N} 1$ virus under test, while the cutoff values for the assay were determined using panels of $\mathrm{WHO}$ and local human serum samples with known antibody levels. Haemagglutination inhibition antibody titres $\geq 1: 40$ were used to define positivity.
Questionnaire data were entered into an Access $^{\circledR}$ database and then exported to SPSS via Excel ${ }^{\circledR}$ spreadsheets. Serology results were provided by the NIC in an Excel spreadsheet, which was then imported into SPSS for analysis.

\section{RESULTS}

Completed questionnaires were received from 1027 healthcare workers out of a workforce of 1476: a response rate of $70 \%$. Blood samples were received from 1005 (98\%) of these responders. Participants' demographic and professional characteristics are shown in Table 1.

\section{Seropositivity}

Seropositivity to influenza A(H1N1)pdm09 was detected in 224 (22\%) of the participants. Younger age, self-reported influenza diagnosis, and receipt of the 2009 seasonal influenza vaccine were associated with seropositivity (Table 2).

\section{Factors associated with seropositivity}

Binary logistic regression was used to test the hypothesis that the recall of one or more influenza-like symptom was predictive of seropositivity to influenza $\mathrm{A}(\mathrm{H} 1 \mathrm{~N} 1) \mathrm{pdm} 09$. The outcome was categorised as either seropositive or seronegative. Of the 1034 participants, this analysis included 976 (94.4\%) who had complete information for all of the factors included in the model. In addition to the experience of symptoms of influenza-like illness, other factors found to significantly affect immunity to influenza A(H1N1)pdm09 from the univariate analysis included: age $1 \leq 45$ years versus 
Table 2. Seropositivity to influenza A(H1N1)pdm09

\begin{tabular}{|c|c|c|c|c|}
\hline & $\begin{array}{c}\text { Seropositive, } \\
n(\%)\end{array}$ & $\begin{array}{c}\text { Seronegative, } \\
n(\%)\end{array}$ & $\begin{array}{l}\text { Odds ratio ( } \\
95 \% \text { CI) }\end{array}$ & $P$-value \\
\hline \multicolumn{5}{|l|}{ Occupation } \\
\hline GPs & $63(21.4)$ & $231(78.6)$ & $1.0(0.7$ to 1.4$)$ & \multirow{3}{*}{0.835} \\
\hline Practice nurses & 90 (23.3) & $297(76.7)$ & $1.1(0.8$ to 1.5$)$ & \\
\hline Receptionists & $71(21.9)$ & $253(78.1)$ & Reference & \\
\hline \multicolumn{5}{|l|}{ Age, years } \\
\hline$<45$ & $91(26.8)$ & 248 (73.2) & 1.5 (1.1 to 2.0$)$ & \multirow[t]{2}{*}{0.016} \\
\hline$>45$ & $133(19.9)$ & $535(80.1)$ & Reference & \\
\hline \multicolumn{5}{|l|}{ Sex } \\
\hline Male & $34(22.7)$ & 116 (77.3) & $1.0(0.7$ to 1.6$)$ & \multirow[t]{2}{*}{0.915} \\
\hline Female & $190(22.2)$ & 667 (77.8) & Reference & \\
\hline \multicolumn{5}{|l|}{ Ethnicity } \\
\hline European & $196(22.0)$ & $693(78.0)$ & $0.9(0.6$ to 1.4$)$ & \multirow[t]{2}{*}{0.725} \\
\hline Other & 28 (23.5) & 91 (76.5) & Reference & \\
\hline \multicolumn{5}{|l|}{ Seasonal vaccine } \\
\hline Vaccinated & $204(23.7)$ & 657 (76.3) & 2.1 (1.3 to 3.4 ) & \multirow[t]{2}{*}{0.003} \\
\hline Not vaccinated & $19(13.0)$ & 127 (87.0) & Reference & \\
\hline \multicolumn{5}{|l|}{ Influenza symptoms } \\
\hline One or more symptoms & $167(23.3)$ & $550(76.7)$ & $1.3(0.9$ to 1.8$)$ & \multirow[t]{2}{*}{0.096} \\
\hline None & 57 (19.3) & $238(80.7)$ & Reference & \\
\hline \multicolumn{5}{|l|}{ Believed had influenza } \\
\hline Yes & 53 (33.5) & 105 (66.5) & $2.0(1.4$ to 2.9$)$ & \multirow[t]{2}{*}{0.0004} \\
\hline No & $171(20.0)$ & $682(80.8)$ & Reference & \\
\hline \multicolumn{5}{|c|}{ Worked in community-based assessment centre } \\
\hline Yes & 47 (23.7) & $151(76.3)$ & $1.1(0.8$ to 1.6$)$ & \multirow[t]{2}{*}{0.503} \\
\hline No & 173 (21.5) & 631 (78.5) & Reference & \\
\hline \multicolumn{5}{|c|}{ Children aged $\leq 18$ years at home } \\
\hline Yes & 104 (22.7) & 355 (77.3) & $1.1(0.8$ to 1.4$)$ & \multirow[t]{2}{*}{0.702} \\
\hline No & $116(21.5)$ & 424 (78.5) & Reference & \\
\hline
\end{tabular}

$>45$ years), participants' belief that they had had influenza that winter, and receipt of seasonal influenza vaccine in 2009. Forward stepwise logistic regression was performed and variables were allowed to remain in the model if statistically significant at $P<0.05$. Table 3 shows the output from the third and final step of the logistic regression model. The final model included three factors: participants' belief that they had had influenza that winter, receipt of the 2009 seasonal influenza vaccine that year, and age.
Participants who believed they had had influenza during the past winter were 1.9 times $(P=0.0005)$ more likely to be seropositive compared to those who did not. Participants who had received the 2009 seasonal influenza vaccination were twice as likely to be seropositive as those who had not been vaccinated $(P=0.008)$. Younger participants ( $\leq 45$ years) were 1.4 times more likely to be seropositive than participants who were older than 45 years $(P=0.029)$.

Asymptomatic infection and time off work Two hundred and twenty-four participants were found to be seropositive, and of these only $53(24 \%)$ recalled having had influenza, and $58(26 \%)$ reported taking time off work.

\section{DISCUSSION}

\section{Summary}

This large serosurvey of front-line primary healthcare workers following the 2009 influenza pandemic found a seroprevalence of $22 \%$ to the influenza A(H1N1)pdm09 virus. Factors associated with seropositivity were: receipt of the 2009 seasonal influenza vaccine, age $<45$ years, and self-reported influenza. Neither employment type, nor working at a community-based assessment centre, was associated with seropositivity. Most participants who had evidence of infection with influenza A(H1N1)pdm09 did not recall having had an influenza-like illness or having taken time off work.

\section{Strengths and limitations}

This is the largest post-pandemic influenza serosurvey of healthcare workers in primary care undertaken or reported. A major strength of this study is that the entire regional primary care workforce was invited to take part, and a high participation rate $(70 \%)$ was achieved.

The serosurvey was completed before vaccination against influenza $A(\mathrm{H} 1 \mathrm{~N} 1)$ pdm09 was available in New Zealand, so all detected immunity is most likely to be due either to infection during the pandemic or pre-existing immunity. However, it is not

\section{Table 3. Logistic regression model of predictors of seropositivity}

\begin{tabular}{lcc} 
Risk factors & $\begin{array}{c}\text { Odds ratio }(\mathbf{9 5 \%} \text { Cl) } \\
\text { for seropositivity }\end{array}$ & \begin{tabular}{c}
$\boldsymbol{P}$-value \\
\hline Recalled having influenza that winter
\end{tabular} \\
\hline Had a seasonal influenza vaccine & $1.9(1.3$ to 2.8$)$ & 0.0005 \\
\hline $\begin{array}{l}\text { Age group, years } \\
\leq 45\end{array}$ & $2.0(1.2$ to 3.3$)$ & 0.0080 \\
$>45$ & $\begin{array}{c}1.4(1.0 \text { to } 1.9) \\
\text { Reference }\end{array}$ & 0.0290 \\
\hline
\end{tabular}


possible to distinguish between participants whose immunity was conferred during the pandemic and those who had preexisting immunity. Pre-pandemic immunity to influenza $A(\mathrm{H} 1 \mathrm{~N} 1) p d m 09$ has been demonstrated in $6.5-7.5 \%$ of New Zealand adults aged $20-59$ years, and in $22.6 \%$ of those aged $>60$ years. ${ }^{26} \mathrm{~A}$ US study found that $34 \%$ of participants born before 1950 had pre-existing immunity to influenza A(H1N1)pdm09.28 In the UK, pre-existing immunity was detected in $9.5 \%$ of those aged $25-49$ years and in $18.5 \%$ of those aged $50-64$ years. ${ }^{29}$

Although data were collected on a range of sociodemographic and workrelated factors, they were not collected on participants' medical histories. These may have affected participants' vaccination history and work patterns during the pandemic, and thus may have acted as confounders in the association observed between receipt of seasonal influenza vaccination and immunity to influenza A(H1N1)pdm09.

The study sample contained only a small number of non-European participants. Other surveys showed higher rates of infection and hospitalisation among Maori and Pacific peoples than among Europeans, ${ }^{3,26}$ so the findings of this study may not be generalisable to these groups.

\section{Comparison with existing literature}

A national New Zealand general population study conducted following the influenza A(H1N1)pdm09 pandemic detected seropositivity in $20-25 \%$ of participants aged $>20$ years, a finding similar to the present one of $22 \% .{ }^{26}$ The general population study included a small sample of 169 primary care workers, of whom $29.6 \%$ were immune to influenza $\mathrm{A}(\mathrm{H} 1 \mathrm{~N} 1) \mathrm{pdm09}$, an estimate that is slightly higher than the present one. A study comparing seropositivity among emergency department healthcare workers and non-healthcare workers in New York found seroprevalence of $21 \%$ among healthcare workers, and no difference in seroprevalence was detected between healthcare workers and non-healthcare workers..$^{30}$ An Australian study similarly found no difference in seroprevalence between clinical and non-clinical tertiary hospital employees; ${ }^{31}$ and a Hong Kong study found no difference in seroprevalence between hospital staff and communitybased blood donors. ${ }^{32}$

A study of healthcare workers and managers in Scottish hospitals found an influenza A(H1N1)pdm09 seroprevalence of $10.3 \%$ at the pandemic peak. ${ }^{33}$ This study did not include primary care staff and may have underestimated total seropositivity, as it was conducted before the end of the pandemic phase. A Singapore study found that staff in an acute hospital had post-pandemic seroprevalence of $7 \%$ (95\% Cl $=5 \%$ to $9 \%$ ). ${ }^{34}$ Other estimates of post-pandemic seroprevalence among the general population range from $11 \%$ to 15\% among English 25-64-year-olds, ${ }^{29}$ to $14 \%$ to $33 \%$ among those aged $>25$ years in Taiwan. ${ }^{35}$

The effect of receipt of seasonal influenza vaccine on the risk of pandemic influenza infection remains uncertain, and studies examining this effect have produced conflicting results. The present study showed an increased risk of seropositivity to influenza A(H1N1)pdm09 among participants who had received previous seasonal influenza vaccination. Similarly, a higher rate of seropositivity to influenza A(H1N1)pdm09 among recipients of seasonal influenza vaccination was reported in another New Zealand study. ${ }^{26}$ This observation may be due to cross-reactivity of antibodies induced by seasonal vaccination to influenza $A(\mathrm{H} 1 \mathrm{~N} 1) p d m 09$, which has been reported, ${ }^{36}$ though is not a consistent finding. ${ }^{37}$ A Canadian observational study reported that receipt of seasonal influenza vaccination was associated with a higher risk of medically attended influenza-like illness, ${ }^{24}$ and it has been suggested that prior receipt of the seasonal vaccine may attenuate the immune response to subsequent infection with pandemic influenza by preventing the induction of heterosubtypic immunity to pandemic strains. ${ }^{38}$ However, the observational design of the Canadian study, like the present one, means that the association may be due to confounding or bias. ${ }^{39} \mathrm{~A}$ protective effect of seasonal vaccination against influenza $\mathrm{A}(\mathrm{H} 1 \mathrm{~N} 1) \mathrm{pdm09}$ infection has been demonstrated in observational studies from Mexico, Argentina, and the US:10-13 while other observational studies, $15,16,40$ and a randomised controlled trial, ${ }^{14}$ have shown no association. A 2012 meta-analysis including 13 studies found no association, but when studies judged to have a moderate or high risk of bias were excluded from the analysis (leaving eight studies), a protective effect on laboratoryconfirmed infection was observed. ${ }^{27}$

\section{Implications for research and practice}

Primary care healthcare workers may be reassured that, following an influenza pandemic, they appeared to be at no greater risk of having been infected las measured by 
seropositivityl than the general population. Furthermore, healthcare workers who had worked at the community-based assessment centres, and hence may have been at greater risk of infection, were no more likely to be seropositive than their colleagues who had not worked in that environment.

Seroprevalence of $22 \%$ following the pandemic meant that the majority of the primary care workforce was still susceptible to the predicted second wave of influenza. Although influenza $A(\mathrm{H} 1 \mathrm{~N} 1)$ pdm09 continued to circulate during the winter of 2010, a pandemic vaccine, and then a seasonal vaccine that included A(H1N1)pdm09, became available to staff. However, this low level of post-pandemic immunity following the first wave may have implications for vaccination policy for the health workforce in the event of future pandemics.

Only $24 \%$ of seropositive participants recalled influenza symptoms, and only $26 \%$ took time off work during the influenza pandemic. Transmission of influenza A(H1N1)pdm09 from asymptomatic individuals has been reported. ${ }^{41}$ This may have implications for healthcare worker vaccination policy ${ }^{42}$ but the risk of asymptomatic influenza transmission remains controversial. ${ }^{43}$

For some healthcare workers, the need to protect themselves and their families may lead them to consider abandoning their workplaces in the event of a pandemic. ${ }^{44}$ Primary healthcare staff faced with the next influenza pandemic may be reassured by the findings of this study that such a response is unnecessary. 


\section{REFERENCES}

1. Centers for Disease Control and Prevention (CDC). Outbreak of swine-origin influenza A (H1N1) virus infection-Mexico, March-April 2009. MMWR Morb Mortal Wkly Rep 2009; 58(17): 467-470

2. World Health Organization. World now at the start of 2009 influenza pandemic; statement to the press by WHO Director-General Dr Margaret Chan. Geneva: WHO, 2009. http://www.who.int/mediacentre/news/statements/2009/h1n1 pandemic_phase6_20090611/en/index.html laccessed 23 Apr 2013).

3. Baker M, Wilson N, Huang Q, et al. Pandemic influenza A(H1N1)v in New Zealand: the experience from April to August 2009. Euro Surveill 2009; 14(34). pii: 19319.

4. Lopez L, Huang Q. Influenza in New Zealand 2009. Client report FW10019. Upper Hutt: Institute of Environmental Science and Research, 2010. http://www.surv. esr.cri.nz/PDF surveillance/Virology/FluAnnRpt/InfluenzaAnn2009 laccessed 23 Apr 2013)

5. Pandemic Influenza Mortality and Morbidity Review Group. Report for the Minister of Health from the Pandemic Influenza Mortality and Morbidity Review Group. Wellington: Ministry of Health, 2010.

6. Williams D, Begg A, Burgess K, et al. Influenza H1N1 2009 in Canterbury: a case study in pandemic response co-ordination. J Prim Health Care 2010: 2(4): 323-329.

7. Chan Y-J, Lee C-L, Hwang S-J, et al. Seroprevalence of antibodies to pandemic (H1N1) 2009 influenza virus among hospital staff in a medical center in Taiwan. $J$ Chin Med Assoc 2010; 73(2): 62-66.

8. Kuster SP, Shah PS, Coleman BL, et al. Incidence of influenza in healthy adults and healthcare workers: a systematic review and meta-analysis. PLoS One 2011 6(10): e26239.

9. Lau LLH, Cowling BJ, Fang VJ, et al. Viral shedding and clinical illness in naturally acquired influenza virus infections. J Infect Dis 2010; 201(10): 15091516

10. Garcia-Garcia L, Valdespino-Gómez JL, Lazcano-Ponce E, et al. Partial protection of seasonal trivalent inactivated vaccine against novel pandemic influenza A/H1N1 2009: case-control study in Mexico City. BMJ 2009; 339: b3928.

11. Echevarría-Zuno S, Mejía-Aranguré JM, Mar-Obeso AJ, et al. Infection and death from influenza A H1N1 virus in Mexico: a retrospective analysis. Lancet 2009. 374(9707): 2072-2079

12. Johns MC, Eick AA, Blazes DL, et al. Seasonal influenza vaccine and protection against pandemic (H1N1) 2009-associated illness among US military personnel. PLoS One 2010; 5(5): e10722.

13. Orellano PW, Reynoso JI, Carlino O, Uez O. Protection of trivalent inactivated influenza vaccine against hospitalizations among pandemic influenza A (H1N1) cases in Argentina. Vaccine 2010; 28(32): 5288-5291.

14. Cowling BJ, $\mathrm{Ng} \mathrm{S}, \mathrm{Ma} \mathrm{ES}$, et al. Protective efficacy of seasonal influenza vaccination against seasonal and pandemic influenza virus infection during 2009 in Hong Kong. Clin Infect Dis 2010; 51(12): 1370-1379.

15. Jefferies S, Earl D, Berry N, et al. Effectiveness of the 2009 seasonal influenza vaccine against pandemic influenza $\mathrm{A}(\mathrm{H} 1 \mathrm{~N} 1) 2009$ in healthcare workers in New Zealand, June-August 2009. Euro Surveill 2011; 16(2). pii: 19761.

16. Centers for Disease Control and Prevention (CDC). Effectiveness of 2008-09 trivalent influenza vaccine against 2009 pandemic influenza A (H1N1) - United States, May-June 2009. MMWR Morb Mortal Wkly Rep 2009; 58(44): 1241-1245.

17. Pebody R, Andrews N, Waight $P$, et al. No effect of $2008 / 09$ seasonal influenza vaccination on the risk of pandemic H1N1 2009 influenza infection in England. Vaccine 2011; 29(14): 2613-2618.

18. Hardelid P, Fleming D, McMenamin J, et al. Effectiveness of pandemic and seasonal influenza vaccine in preventing pandemic influenza A(H1N1)2009 infection in England and Scotland 2009-2010. Euro Surveill 2011; 16(2). pii: 19763.

19. Puig-Barberà J, Arnedo-Pena A, Pardo-Serrano F, et al. Effectiveness of seasonal 2008-2009, 2009-2010 and pandemic vaccines, to prevent influenza hospitalizations during the autumn 2009 influenza pandemic wave in Castellón, Spain. A test-negative, hospital-based, case-control study. Vaccine 2010; 28(47): 7460-7467.

20. Kelly HA, Grant KA, Fielding JE, et al. Pandemic influenza H1N1 2009 infection in Victoria, Australia: no evidence for harm or benefit following receipt of seasonal influenza vaccine in 2009. Vaccine 2011; 29(37): 6419-6426.

21. Valenciano M, Kissling E, Cohen JM, et al. Estimates of pandemic influenza vaccine effectiveness in Europe, 2009-2010: results of Influenza Monitoring Vaccine Effectiveness in Europe (I-MOVE) multicentre case-control study. PLoS
Med 2011; 8(1): e1000388

22. Nelson CA, France EK, Shetterly SM, Glanz JM. Seasonal influenza vaccination status among children with laboratory evidence of pandemic $\mathrm{H} 1 \mathrm{~N} 1$ infection. Pediatr Infect Dis J 2011; 30(7): 562-565.

23. Carcione D, Giele C, Goggin L, et al. Association between 2009 seasonal influenza vaccine and influenza-like illness during the 2009 pandemic: preliminary results of a large household transmission study in Western Australia. Euro Surveill 2010; 15(28). pii: 19616.

24. Skowronski DM, De Serres G, Crowcroft NS, et al. Association between the 2008-09 seasonal influenza vaccine and pandemic H1N1 illness during springsummer 2009: four observational studies from Canada. PLoS Med 2010; 7(4): e1000258

25. Janjua NZ, Skowronski DM, Hottes TS, et al. Seasonal influenza vaccine and increased risk of pandemic $\mathrm{A} / \mathrm{H} 1 \mathrm{N1} 1$-related illness: first detection of the association in British Columbia, Canada. Clin Infect Dis 2010; 51(9): 1017-1027.

26. Bandaranayake D, Huang QS, Bissielo A, et al. Risk factors and immunity in a nationally representative population following the 2009 influenza A(H1N1] pandemic. PLoS One 2010; 5(10): e13211.

27. Yin JK, Chow MYK, Khandaker G, et al. Impacts on influenza A(H1N1)pdm09 infection from cross-protection of seasonal trivalent influenza vaccines and A(H1N1)pdm09 vaccines: systematic review and meta-analyses. Vaccine 2012; 30(21): 3209-3222

28. Hancock K, Veguilla V, Lu X, et al. Cross-reactive antibody responses to the 2009 pandemic H1N1 influenza virus. N Engl J Med 2009; 361(20): 1945-1952.

29. Miller $E$, Hoschler $K$, Hardelid $P$, et al. Incidence of 2009 pandemic influenza A H1N1 infection in England: a cross-sectional serological study. Lancet 2010; 375(9720): 1100-1108.

30. Alagappan K, Silverman RA, Hancock K, et al. Seropositivity for influenza A(H1N1)pdm09 virus among front-line health care personnel. Emerg Infect Dis 2013; 19(1): 140-143.

31. Marshall C, Kelso A, McBryde E, et al. Pandemic (H1N1) 2009 risk for frontline health care workers. Emerg Infect Dis 2011; 17(6): 1000-1006.

32. Zhou Y, Ng DM, Seto WH, et al. Seroprevalence of antibody to pandemic influenza A (H1N1) 2009 among healthcare workers after the first wave in Hong Kong. J Hosp Infect 2011; 78(4): 308-311.

33. Smith $K$, Warner $P$, Williams LJ, et al. Prevalence of influenza A (H1N1) seropositivity in unvaccinated healthcare workers in Scotland at the height of the global pandemic. J Environ Public Health 2011; 2011: 407505

34. Chen MI, Lee VJ, Lim WY, et al. 2009 Influenza A(H1N1) seroconversion rates and risk factors among distinct adult cohorts in Singapore. JAMA 2010; 303(14): 1383-1391.

35. Chen CJ, Lee PI, Chang SC, et al. Seroprevalence and severity of 2009 pandemic influenza A H1N1 in Taiwan. PLoS One 2011; 6(9): e24440.

36. Xie H, Jing X, Li X, et al. Immunogenicity and cross-reactivity of 2009-2010 inactivated seasonal influenza vaccine in US adults and elderly. PLoS One 2011; 6(1): $\mathrm{e} 16650$.

37. Uno S, Kimachi K, Matsuo F, et al. Cross-reactive antibody response to the pandemic A (H1N1) 2009 influenza virus induced by vaccination with a seasonal trivalent influenza vaccine: a longitudinal study of three influenza seasons in Japan. Microbiol Immunol 2012; 56(12): 810-816.

38. Bodewes R, Kreijtz JHCM, Rimmelzwaan GF. Yearly influenza vaccinations: a double-edged sword? Lancet Infect Dis 2009; 9(12): 784-788

39. Viboud C, Simonsen L. Does seasonal influenza vaccination increase the risk of illness with the 2009 A/H1N1 pandemic virus? PLoS Med 2010; 7(4): e1000259.

40. Kelly H, Grant K. Interim analysis of pandemic influenza (H1N1) 2009 in Australia: surveillance trends, age of infection and effectiveness of seasonal vaccination. Euro Surveill 2009; 14(31). pii: 19288

41. Gu Y, Komiya N, Kamiya H, et al. Pandemic (H1N1) 2009 transmission during presymptomatic phase, Japan. Emerg Infect Dis 2011; 17(9): 1737-1739.

42. Poland GA, Tosh $P$, Jacobson RM. Requiring influenza vaccination for health care workers: seven truths we must accept. Vaccine 2005; 23(17-18): 2251-2255.

43. Patrozou E, Mermel LA. Does influenza transmission occur from asymptomatic infection or prior to symptom onset? Public Health Rep 2009; 124(2): 193-196.

44. Ehrenstein B, Hanses F, Salzberger B. Influenza pandemic and professional duty: family or patients first? A survey of hospital employees. BMC Public Health 2006; 6: 311. 